\title{
Studying the Stability of one of Non-Linear Autoregressive Models with
}

\section{Application}

\section{Abdulghafoor Salim}

drabdul_salim@uomosul.edu.iq

College of Computer Science and Mathematics,

Received on: 29/3/2011

\author{
Raad Awad Alhamdani \\ College of Education \\ University of Tikrit,
}

\section{Accepted on: 16/5/2011}

In This paper we suggest one of the models for non-linear Autoregressive by using local linear approximation method, then we studied the conditions of stationarity of the model specifically the conditions of stationarity the non-zero singular point of the model. And the conditions for stationarity to limit cycle stationarity.

Conditions are applied in the aforementioned theories on data represent the monthly average wind speed in model of Baghdad city. The samples are classified by using one of non-linear Autoregressive model the findings are the value of non-zero fixed point and the conditions of suggested stationarity model .

Keywords: Stability, non-linear autoregressve.

$$
\begin{aligned}
& \text { دراسة استقرارية احد نماذج الانحدار الذاتي غير الخطي مع تطبيق } \\
& \text { رعد عواد حميد } \\
& \text { عبد الغفور جاسم سالم } \\
& \text { كلية التربية، جامعة تكربت }
\end{aligned}
$$

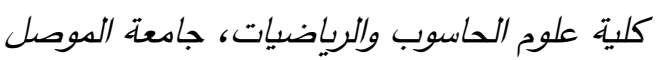

تاريخ قبول البحث: 16 / 5 / 2011

\section{الملخص}

تم في هذه البحث اقتراح احد النماذج للانحدار الذاتي غير الخطي باستخدام طريقة التقريب بالخطية

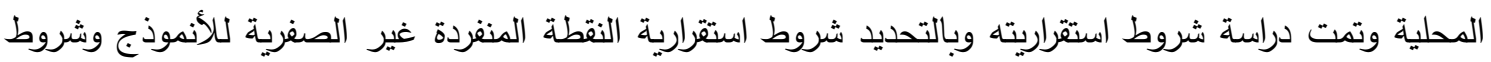
استقرارية دورة النهاية. لقد تم تطبيق الشروط الموجودة في المبرهنتين المذكورتين آنفاً على بيانات تمثل المعدل الثهري لسرعة الرياح في مدينة بغداد وتم نمذجة هذه البيانات باستخدام احد نماذج الانحدار الذاتي غير الخطي

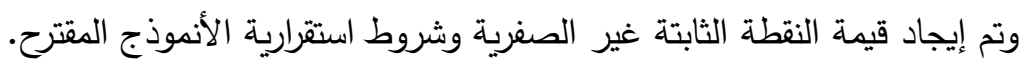

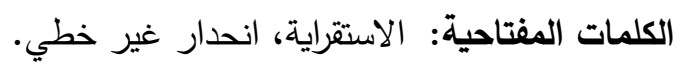
1- 1 المقدمة

إن دراسة تحليل السلاسل الزمنية هو موضوع واسع, وقد ازداد اتساعه في العقود الأخيرة بثكل كبير نظراً لكثرة تطبيقاته في الاتصالات والهندسة والأنواء الجوية ل... الخ. إن الهدف الأساسي من بناء أنموذج سلسلة زمنية

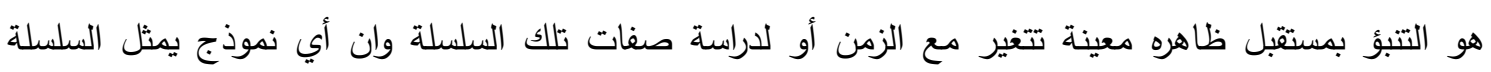
الزمنية يجب أن يتصف بصفات محددة لكي يعطي تتبؤات صحيحة إلى حد معين وأهم هذه الصفات هي

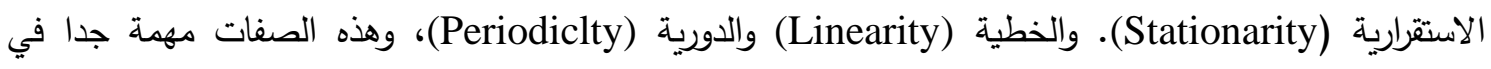

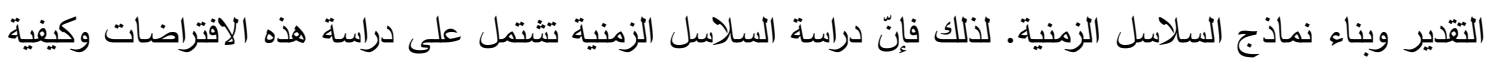

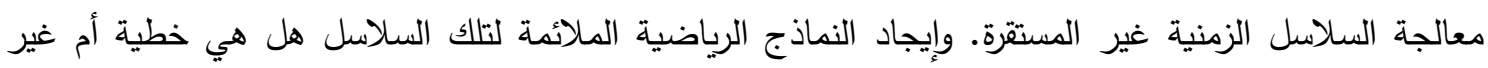

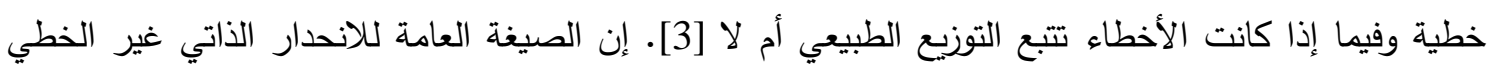
من الرتبة p يمكن التعبير عنه بالثكل الأتي: 
$X_{t}=f\left(x_{t-1}, x_{t-2}, \ldots, x_{t-p}\right)+Z_{t}$

(.) هي دالة غير خطية و $f$ (White Noise) وهناك دراسات عديدة في

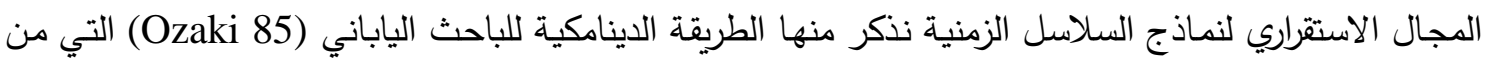

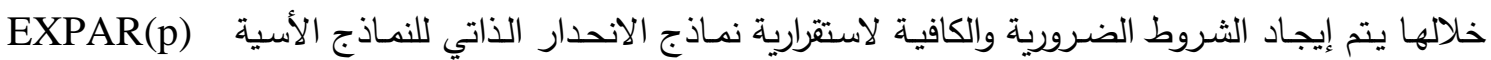
(Kurt Hornik, وكذلك دراسـة الاستقرارية في الانحدار الذاتي للشبكات عام (1999) من قبل الباحثين Fredrick, Adrrcn And Trapletti) الاستقرارية في النمـاذج غير الخطيـة من الدرجـة الأولى. وفي عـام (2005) قام الباحث عباس وسـالم بدراسـة استقرارية النموذج اللوجستي LSTAR(P) [1]. وفي (2010) درس الباحث سالم وعواد استقرارية بعض النماذج

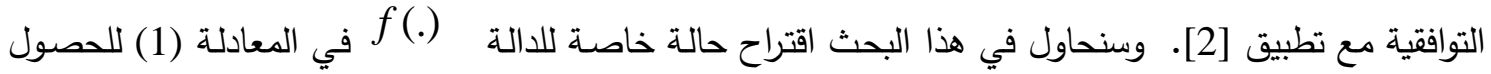
على احد نمـاذج الانحدار الذاتي غير الخطي ومن ثم دراسـة استقراريته بطريقة التقريب غير الخطي. ونحاول تطبيق النتائج التي حصلنا عليها على السلسلة الزمنية لسرعة الرياح في مدينة بغداد.

2- هدف البحث:

يهدف البحث إلى اقتراح أنموذج انحدار ذاتي غير خطي ومن ثم دراسة استقراريته باستخدام طريقة التقريب بالخطية

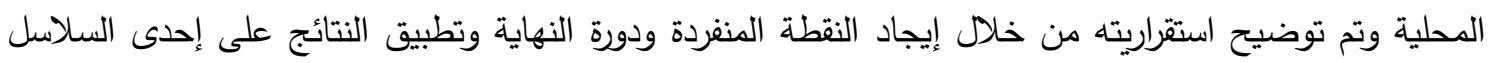
الزمنية التي تمتلك صفات دورية.

3- 3فاهيم ومبادئ أساسية:

[5][4] (Stationarity) 1 - 1 : الاستقرارية

تكون السلسلة الزمنية $\left\{X_{t}\right.$ مستقرة إذا كانت في حالة موازنة إحصائية، أي أنّ خصائصها الإحصائية لا تتأثرّ بالزمن، وتكون السلسلة Strictly Stationary للمتغيرات النقاط الزمنية المختارة

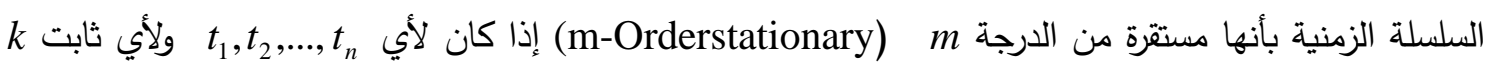
فان جميع العزوم من الدرجة $m$ الـ $E\left[\left\{X_{t_{1}}\right\}^{m_{1}},\left\{X_{t_{2}}\right\}^{m_{2}}, \ldots,\left\{X_{t_{n}}\right\}^{m_{n}}\right]=E\left[\left\{X_{t_{1+k}}\right\}^{m_{1}},\left\{X_{t_{2+k}}\right\}^{m_{2}}, \ldots,\left\{X_{t_{n+k}}\right\}^{m_{n}}\right]$ المختارة . $m_{1}+m_{2}+\ldots+m_{n} \leq m$

ولحالة خاصة يقال إن السلسلة الزمنية $\left\{X_{t}\right.$ أنها مستقرة من الرتبة الأولى (First Order Stationary) إذا $E\left(X_{t}\right)=\mu$ كانت كما يقال للسلسلة الزمنية $\}$ كان الآتية: 1) $E\left(X_{t}\right)=\mu$ ر كمية ثابتة لا تعتمد على 
2) $\operatorname{Var}\left(X_{t}\right)=\sigma_{x}^{2}$

$t$ t $\sigma_{x}^{2}$

3) $\operatorname{Cov}\left[X_{t_{1}}, X_{t_{2}}\right]=\gamma_{\left(t_{1}, t_{2}\right)}$

دالة بذلالة |

فالاستقرارية مفهوم عام في الأنظمة الديناميكية (الحركية) التي تتكون من نظام من المعادلات التفاضلية أو الفرقية

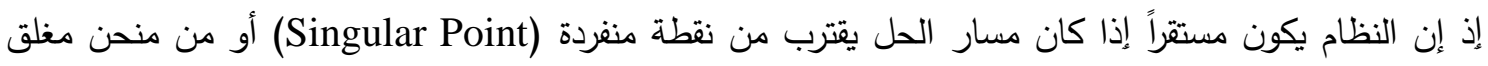
يطلق عليه دورة النهاية (Limit Cycle).

Autoregressive Model of Order p: AR(p):p 2 - 2 : نموذج الاتحدار الذاتي من الرتبة دوة يمكن تعريف أنموذج الانحدار الذاتي من الرتبة p للسلسلة الزمنية المستقرة $X_{t}=\phi_{1} X_{t-1}+\ldots+\phi_{p} X_{t-p}+Z_{t}$

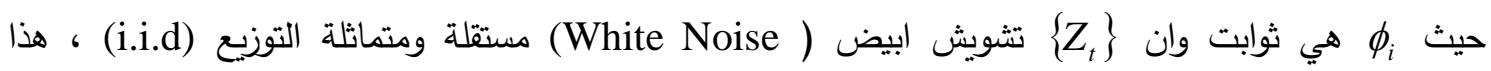
الأنموذج يعبر عن العلاقة بين حاضر السلسلة

2-3 : نموذج الاتحدار الذاتي غير الخطي المقترح:

$X_{t}=1-\frac{1}{1+\phi X_{t-1}}+Z_{t}$ ليكن لدينا أنموذج الانحدار الذاتي الأتي:

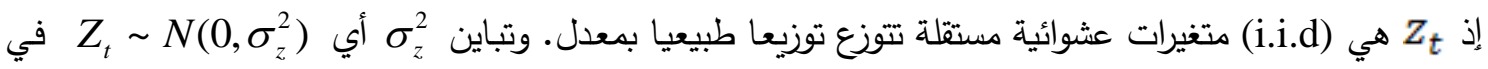
الزمن المتقطع، والمعلمة و هي ثابت حقيقي.

[10] [9-3

النقطة المنفردة ك للأنموذج (1) تعرف بأنها تلك النقطة التي تحقق الثرط الآتي: إن أي مسار للنموذج (1) يبدأ من نقطة قريبة بشكل كاف من ك ك يقترب منها إمـا عندما

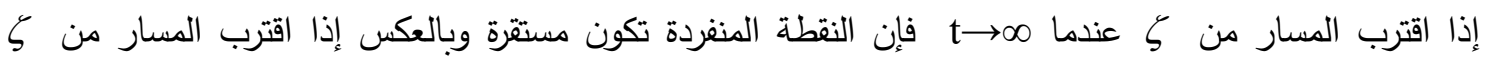

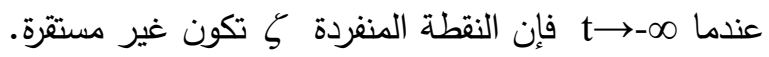
[10] [9] 4 - 3

دورة النهاية للنموذج (1) تعرف بأنها المسار المغلق والمعزول عدد صحيح موجب. المقصود بأنَّ المسار مغلق هو أنه إذا كانت القيم الابتدائية q تنتمي إلى دورة النهاية فإن

$$
\text { . } k \text { لأي عدد صحيح }\left(x_{1}, x_{2}, x_{3}, \ldots, x_{p}\right)=\left(x_{1+k q}, x_{2+k q}, x_{3+k q}, \ldots, x_{p+k q}\right)
$$

والمقصود بالمعزول (Isolated) إن أي مسار يبدأ قريباً من دورة النهاية بثكل كاف يتقارب نحو دورة

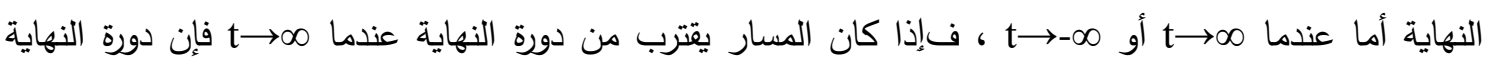
تكون مستقرة وبالعكس إذا كان المسار يقترب من دورة النهاية عندما م-ه t فإن دورة النهاية تكون غير مستقرة.

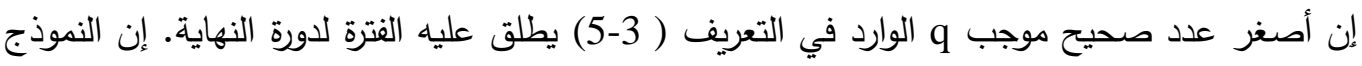
(3) يحوي على نقطة منفردة صفرية ونقاط منفردة أخرى غير صفرية ( Non-Zero Singular Points) 


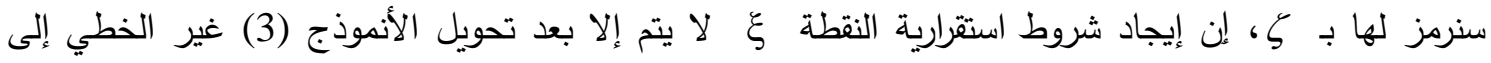
نموذج انحدار ذاتي خطي باستخدام تقنية التقريب بالخطية المحلية وفي جوار النقطة ظ. لقد لقد استخدم الباحث Ozaki

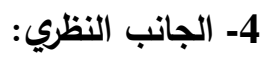

\section{4 - 1 الاستقرارية حسب طريقة اوزاكي:}

سنحاول في هذا المبحث دراسة الاستقرارية للأنموذج المقترح معادلة رقم (3). من الواضح أن معامل الانحدار يعتمد على | بنى

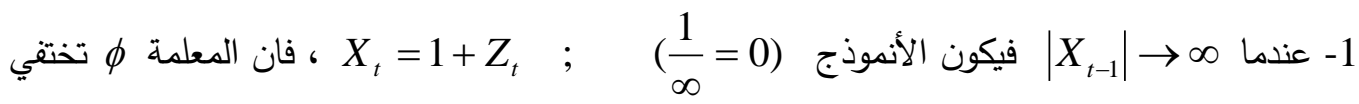
وكذلك

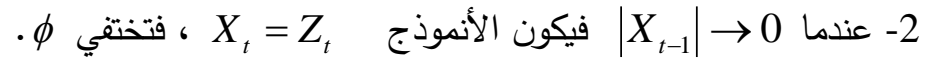

سنحاول في هذا المبحث دراسة استقرارية الأنموذج المقترح باستخدام التقريب بالمحلية غير الخطية وتتلخص

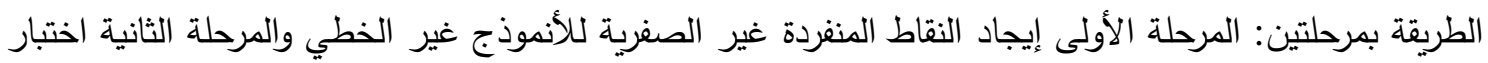
استقرارية تلك النقطة من خلال دورة النهاية [10] لدراسة الشروط الخاصة باستقرارية الأنموذج (3) نجد النقطة المنفردة غير الصفرية وناهة

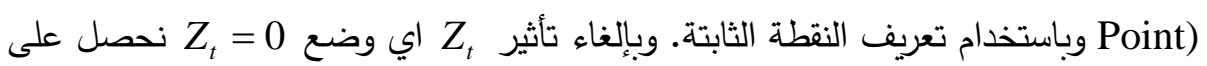
$\xi=1-\frac{1}{1+\phi \xi} \Rightarrow \xi(\phi \xi+(1-\phi))=0$

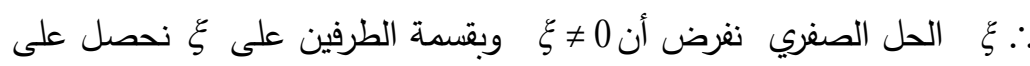

$\phi \xi+(1-\phi)=0$

$\therefore \xi=1-\frac{1}{\phi}$

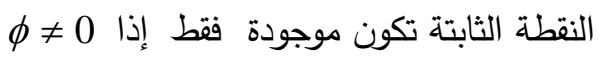
المبرهنة الآتية تبين شروط الاستقرارية للأنموذج (3) بالاعتماد على ثوابت للأنموذج هود وكما يلي: [10] [9] : 3 : 3

النقطة المنفردة غير الصفرية (إن وجدت) للأنموذج (3) مستقرة إذا كانت جذور المعادلة $\lambda^{p}-\sum_{i=1}^{p} h_{i} \lambda^{p-i}=0$ 
البرهان: نأخذ جوار مفتوح حول $\xi$ ( حيث $\xi$ نقطة منفردة غير صفرية ) بنصف قطر صغيرة كفاية بحيث أن المعادلة البينية (Variational Equation). ضع النموذج (3) بالثكل

$$
\begin{aligned}
& \xi+\xi_{t}=1-\frac{1}{1+\phi\left(\xi+\xi_{t-1}\right)} \\
& \left(1-\frac{1}{\phi}\right)+\xi_{t}=1-\frac{1}{1+\phi\left(\left(1-\frac{1}{\phi}\right)+\phi \xi_{t-1}\right)}
\end{aligned}
$$$$
\left(1-\frac{1}{\phi}\right)+\xi_{t}=1-\frac{1}{1+\phi-1+\phi \xi_{t-1}}
$$$$
\xi_{t}=\frac{\phi \xi_{t-1}}{\phi^{2}\left(1+\xi_{t-1}\right)}
$$$$
\xi_{t}=\frac{\xi_{t-1}}{\phi\left(1+\xi_{t-1}\right)}
$$$$
\xi_{t}=\frac{\xi_{t-1}\left(1-\xi_{t-1}\right)}{\phi\left(1+\xi_{t-1}\right)\left(1-\xi_{t-1}\right)}
$$$$
\xi_{t}=\frac{\xi_{t-1}-\xi_{t-1}^{2}}{\phi\left(1-\xi_{t-1}^{2}\right)}
$$

بما أن

$$
\xi_{t}=\frac{1}{\phi} \xi_{t-1}
$$

$$
\phi \neq 0 \text { بحيث }
$$

وبما أن الأنموذج الذي حصلنا علية هو أنموذج الانحدار الذاتي الخطي هو AR(1) فانه يكون مستقرا إذا كانت

جذور المعادلة المميزة اقل من الواحد أي أن 1> $\left|\frac{1}{\phi}\right|$ حيث لدراسة وإيجاد شروط استقرارية دورة النهاية (إن وجدت) للأنموذج (3) نجد أولا شروط استقرارية الأنموذج المذكور والمبرهنة الآتية تعطي الثروط بدلالة معلمات النموذج.

[10] [9] : 4 - 4

تكون دورة النهاية بالدورة qللأنموذج (3) مستقرة مدارياً إذا تحقق الثرط

$\left|\prod_{i=1}^{q}\left[\frac{\phi_{i}}{1+\phi_{i} X_{t+q-i}}\right]\right|<1$ 
البرهان: نفرض أن الأنموذج $X_{t}, X_{t-1}, X_{t+2}, \ldots ., X_{t+q}=X_{t}$

$$
\begin{aligned}
& X_{t}+\xi_{t}=1-\frac{1}{1+\phi\left(X_{t-1}+\xi_{t-1}\right)} \\
& X_{t}+\xi_{t}=\frac{1+\phi\left(X_{t-1}+\xi_{t-1}\right)-1}{1+\phi\left(X_{t-1}+\xi_{t-1}\right)} \\
& X_{t}+\xi_{t}=\frac{1+\phi X_{t-1}+\phi \xi_{t-1}-1}{1+\phi\left(X_{t-1}+\xi_{t-1}\right)} \\
& X_{t}+\xi_{t}=\frac{1+\phi X_{t-1}-1+\phi \xi_{t-1}}{1+\phi\left(X_{t-1}+\xi_{t-1} \frac{\xi_{t-1}}{\xi_{t-1}}\right)}
\end{aligned}
$$$$
X_{t}+\xi_{t}=\frac{1+\phi X_{t-1}-1+\phi \xi_{t-1}}{1+\phi\left(X_{t-1}+\frac{\xi_{t-1}^{2}}{\xi_{t-1}}\right)}
$$

بما أن ف

$$
X_{t}+\xi_{t}=\frac{1+\phi X_{t-1}-1+\phi \xi_{t-1}}{1+\phi X_{t-1}}
$$$$
X_{t}+\xi_{t}=\frac{1+\phi X_{t-1}-1}{1+\phi X_{t-1}}+\frac{\phi \xi_{t-1}}{1+\phi X_{t-1}}
$$$$
X_{t}+\xi_{t}=1-\frac{1}{1+\phi X_{t-1}}+\frac{\phi \xi_{t-1}}{1+\phi X_{t-1}}
$$

$$
X_{t}=1-\frac{1}{1+\phi X_{t-1}}
$$

$$
\begin{aligned}
& X_{t}+\xi_{t}=X_{t}+\frac{\phi \xi_{t-1}}{1+\phi X_{t-1}} \\
& \xi_{t}=\frac{\phi \xi_{t-1}}{1+\phi X_{t-1}} \\
& \xi_{t}=\left(\frac{\phi}{1+\phi X_{t-1}}\right) \xi_{t-1} \\
& \frac{\xi_{t}}{\xi_{t-1}}=\left(\frac{\phi}{1+\phi X_{t-1}}\right)
\end{aligned}
$$




$$
\begin{aligned}
& T\left(X_{t-1}\right)=\left(\frac{\phi}{1+\phi X_{t-1}}\right) \\
& \Rightarrow \xi_{t}=T\left(X_{t-1}\right) \xi_{t-1} \\
& \therefore \xi_{t+q}=T\left(X_{t+q-1}\right) \xi_{t+q-1} \\
& =T\left(X_{t+q-1}\right) T\left(X_{t+q-2}\right) \xi_{t+q-2} \\
& =T\left(X_{t+q-1}\right) T\left(X_{t+q-2}\right) \ldots \ldots . T\left(X_{t}\right) \xi_{t} \\
& \xi_{t+q}=\left|\prod_{i=1}^{q} T\left(X_{t+q-i}\right)\right| \xi_{t} \\
& T\left(s_{t}\right)=\frac{\phi}{1+\phi s_{t-1}} \text {. } \\
& \left|\frac{\xi_{t+q}}{\xi_{t}}\right|=\left|\prod_{i=1}^{q} T\left(X_{t+q-i}\right)\right| \\
& \text { أي أن } \\
& \text { وبالتالي فان :- } \\
& \text { ولأجل التقارب نحو الصفر يجب أن تكون هذه النسبة اقل من واحد أي أن } \\
& \left|\prod_{i=1}^{q} T\left(X_{t+q-i}\right)\right|<1 \\
& \left|\prod_{i=1}^{q}\left[\frac{\phi_{i}}{1+\phi_{i} X_{t+q-i}}\right]\right|<1
\end{aligned}
$$

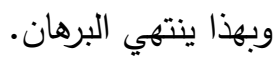

\section{5plication الجانب التطبيقي}

Introduction 1 - 1 : المقدمة

في هذه الفقرة يتم تطبيق شروط استقرارية الأنموذج (3) والتي تم إيجادها في الفقرة السابقة وبالتحديد استقرارية النقطة المنفردة غير الصفرية للأنموذج التي تم وضعها في القضية (4-1) واستقرارية دورة النهاية بالنسبة

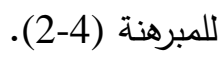
أن البيانات المستخدمة في هذا التطبيق هي بيانات سرعة الرياح في مدينة بغداد إذ أن سرعة الرياح تزداد في فصل الثتاء وتقل في فصل الصيف. تم استخدام البرنامج الجاهز (Statistical V.6) بنسخته السادسة في تخمين قيم معلمات النماذج التي تم إيجادها في هذا البحث وبالتحديد تم استخدام جزء البرنامج الخاص بالتخمين اللاخطي ( Nonlinear Estimation 
Description of Data 5

إنَّ التسجيلات الثهرية لسرعة الرياح في مدينة بغداد أو بشكل أدق السلسلة الزمنية لسرعة الرياح في

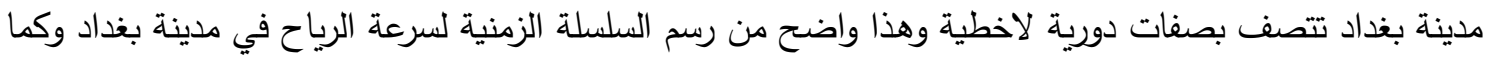

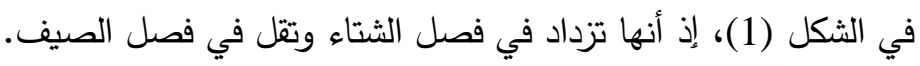
Plot of variable: VAR1

$\mathrm{x}+0$

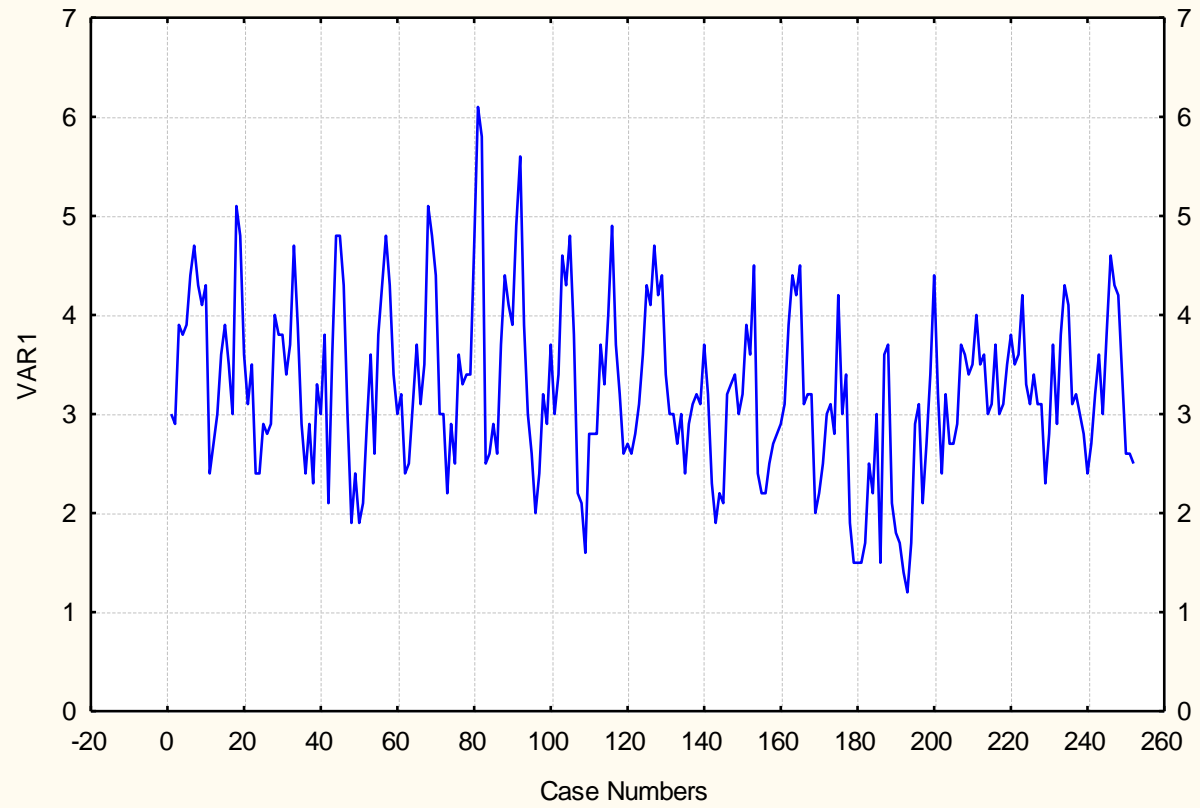

الثكل (1) : رسم السلسلة الزمنية لبيانات معدلات سرعة الرياح في مدينة بغداد للفترة من (1970-1990)

5 - 5 : نمذجة السلسلة الزمنية لسرعة الرياح في مدينة بغداد باستخدام نماذج NOLAR(1) في هذه الفقرة تمت نمذجة السلسلة الزمنية الثهرية لمعدلات سرعة الرياح في مدينة بغداد، وباستخدام برنامج Statistical V.6 وحصلنا على النموذج الأتي: $X_{t}=1-\frac{1}{1+114889.1 X_{t-1}}+Z_{t}$

$$
\text { وبتباين بواقي } 0.732189
$$

$\operatorname{AIC}(p)=2.270754$

حيث AIC تعرف بالثكل

$\operatorname{AIC}(p)=-2 \ln \left(\sigma_{z}^{2}\right)+2 M$

وان P تمثل رتبة الأنموذج وM عدد معلمات الأنموذج المخمن و فهو رسم الاحتمالية الطبيعية لبواقي الأنموذج (1) NOLAR . 


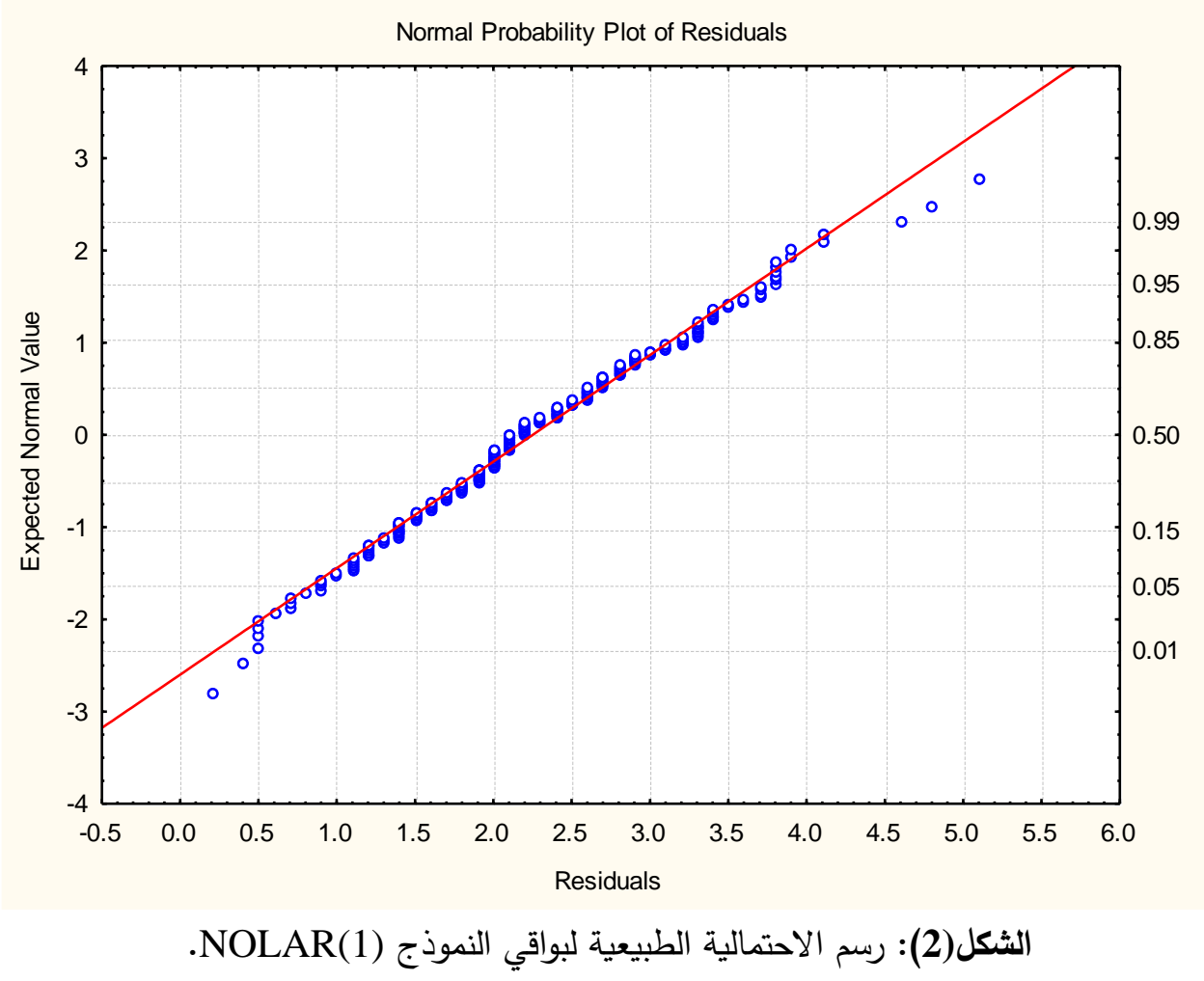

إن البواقي هنا تمثل التشويش الأبيض الذي يفترض به أن يتوزع توزيعاً طبيعياً بتباين

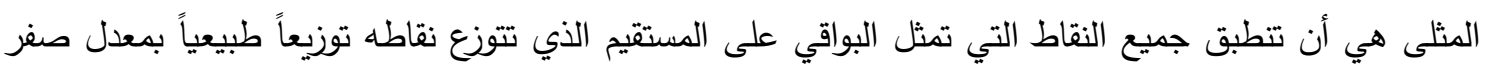
وتباين

باستخدام العلاقة (4) تم حساب قيمة النقطة المنفردة غير الصفرية للنموذج ووجد بأن هناك قيمة النقطة المنفردة هي 0.99999913= و و وباستخدام العلاقة (5) نحصل على المعادلة الفرقية الآتية $\xi_{t}=0.00000007 \xi_{t-1}$

وواضح أن النقطة المنفردة غير الصفرية للأنموذج مستقرة لأنَّ جذور المعادلة المميزة $\lambda-0.00000007=0$

ذات قيمة مطلقة أقل من الواحد وبالتالي يكون أنموذج NOLAR(1 مستقرا حسب المبرهنة (4-3). 6- - 6 الاستنتاجات والتوصيات:

من خلال دراستتا للاستقرارية باستخدام تقنية التقريب الخطية لأحد النماذج غير الخطية المقترح تبين لنا: 1- هناك نقطتان منفردتان وكان احدهما النقطة الصفرية (Trivial والنقطة الثابتة ( Solution) - $\left|\frac{1}{\phi}\right|<1$ كانت 2- الأنموذج يمتلك دورة نهاية وهذا ما تم برهانه باستخدام المبرهنة (4-2). 
3- ومن خلال التطبيق تبين ان سرعة الرياح دورية وتمتلك نقطه منفردة مستقرة حيث جذور المعادلة المميزة

$$
\text { للنموذج يحقق العلاقة 1> } 1 \text { > ل }
$$

ويمكن الاستفادة من الطريقة (طريقة التقريب الخطية) لدراسة نماذج غير خطية أكثر تعقيدا وباستخدام توسيع تايلر والمبرهنات أنفة الذكر في بحثنا. 


$$
\begin{aligned}
& \text { المصادر } \\
& \text { عباس، أزهر وسالم، عبد الغفور جاسم (2007)، "استقرارية نموذج الانحدار الذاتي اللوجستي"، مجلة }
\end{aligned}
$$

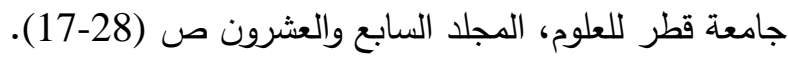

$$
\begin{aligned}
& \text { سـالم، عبد الغفور جاسم وحميد رعد عواد، (2010)، "دراسـة استقرارية بعض النمـاذج التوافقيـة مـع }
\end{aligned}
$$

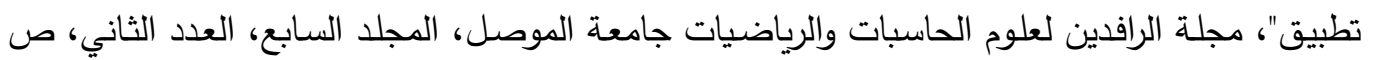

$$
\begin{aligned}
& \text {.(157-139) } \\
& \text { المهداوي، هيفاء جعفر، (1999)، "الانحدار الذاتي غير المستقر ذو الدرجات الدنيا"، أطروحة دكتوراه، } \\
& \text { كلية الإدارة والاقتصاد، الجامعة المستتصرية. }
\end{aligned}
$$

[4] Chatfield, (1978), "The analysis of time series: Theory Practice", Chapman and Hall, London.

[5] Chatfield, Christopher, (1984), "The analysis of time series: 4th Edition Chapman and Hall.

[6] Chatfield, C., (1984), "The Analysis of Time Series An Introduction", 3rd Ed., J. W. Arrow Smith Ltd., Bristol, GB.

[7] Fonseca, G., (2000), "stability and Estimation of Non-linear Time series models", Un put. D. Thesis, University of Padura, Italy.

[8] Friedrich leisch and Kurt Hornik, (1999), "stationarity and stability of Autoregressive Neurel Network processes", MIT Prss, Cambridge, MA,USA.

[9] Ozaki, T., (1982), "The Statistical Analysis of Perturbed Limit Cycle Processes Using Nonlinear Time series Models", Journal of Time Series Analysis, V. 3, No. 1, pp. 29-41.

[10] Ozaki, T., (1985), "Nonlinear Time Series Models and Dynamical Systems", Handbook of Statistics, V. 5 (Ed. Hannan , E. J. and Krishnailah , P. R. and Rao, M.M.), Elsevier Science Publishers B.V., pp. 25-83. 